\title{
NUMERICAL APPROXIMATION OF MULTI-PHASE PENROSE-FIFE SYSTEMS
}

\author{
CARSTEN GRÄSER, MAX KAHNT, AND RALF KORNHUBER
}

\begin{abstract}
We consider a non-isothermal multi-phase field model. We subsequently discretize implicitly in time and with linear finite elements. The arising algebraic problem is formulated in two variables where one is the multi-phase field, and the other contains the inverse temperature field. We solve this saddle point problem numerically by a non-smooth Schur-Newton approach using truncated non-smooth Newton multigrid methods. An application in grain growth as occurring in liquid phase crystallization of silicon is considered.
\end{abstract}

\section{INTRODUCTION}

The mathematical modelling of phase transitions has a long history and has stimulated new developments in the field of variational inequalities and free boundary value problems over more than three decades $[3,18,36]$. Particular attention was paid to problems of Stefan-type [48] and their mathematical description by phase field models [17]. In this approach, phase transitions are represented by an order parameter that is strongly varying across the (diffuse) interface. The evolution of the order parameter is typically obtained from some gradient flow of a suitable Ginzburg-Landau free energy that provides non-decreasing entropy (thermodynamical consistency) and could be mass conserving (phase separation) or non-conserving (phase transition). More recently Stinner et al. [24] extended well-established thermodynamically consistent, two-phase Penrose-Fife models [17] to multiple phases (non-conserved) and components (conserved). Existence of solutions to the resulting balance equations for the energy, order parameters, and concentrations of components was studied in [47].

While the numerical analysis of two-phase Penrose-Fife models was based on implicit time discretization [37], previous numerical computations with multiple phases and components were typically based on an explicit approach [42]. In this way, the solution of non-smooth, large-scale algebraic systems is avoided at the expense of severe stability constraints on the time step.

In this paper, we consider a multi-phase extension of the classical PenroseFife system [17, 37, 45]. Following Stinner et al. [24], this system is derived from a general entropy functional that combines a Ginzburg-Landau energy with the thermodynamic entropy. We concentrate on a numerical approach based on semi-implicit time discretization (with explicit treatment of the concave terms [19, 28]) and first-order Taylor approximation of nonlinearities associated with inverse temperature. Variational arguments are used to show the existence and uniqueness of solutions of the resulting spatial problems and the thermodynamic consistency of this time discretization. Spatial discretization is performed by piecewise linear finite elements with adaptive mesh refinement based on hierarchical a posteriori error estimation $[30,27]$. The resulting large-scale non-smooth algebraic systems are solved by non-smooth Schur-Newton multigrid (NSNMG) methods [25, 29, 31] exploiting again the saddle point structure of these problems. In our numerical

Date: October 16, 2018. 
experiments, we observe optimal order of convergence of the spatial discretization and mesh-independent, fast convergence speed of NSNMG with nested iteration. Furthermore, our computations suggest that non-decreasing entropy is preserved under the spatial discretization. Application to a liquid phase crystallization (LPC) process occurring in the fabrication of thin film silicon solar cells [41] underline the potential of the presented solution approach.

\section{Phase FIELd MODELling}

2.1. Thermodynamical background. Let $\Omega \subset \mathbb{R}^{d}, d=1,2,3$, be a bounded domain with Lipschitz boundary $\Gamma=\partial \Omega$. Following [24], we consider the entropy functional

$$
S(e, \phi)=\int_{\Omega} s(e, \phi)-\left(\frac{\varepsilon}{2} \gamma^{2}(\phi, \nabla \phi)+\frac{1}{\varepsilon} \psi(\phi)\right) \mathrm{d} x,
$$

where the entropy density $s$ depends on the internal energy density $e$ and on the multi-phase field $\phi=\left(\phi_{\alpha}\right)_{\alpha=1}^{M}, \gamma$ represents the surface gradient entropy, and $\psi$ a multi-well potential with $M$ distinct minima. The components of $\phi$ describe relative fractions of a given substance. Hence, it is natural to impose the algebraic constraint

$$
\sum_{\alpha=1}^{M} \phi_{\alpha}=1
$$

We postulate the Gibbs relation $[1,24]$

$$
\mathrm{d} f=-s \mathrm{~d} T+\sum_{\alpha=1}^{M} f_{, \phi_{\alpha}} \mathrm{d} \phi_{\alpha}
$$

with absolute temperature $T>0$ and a Helmholtz free energy density $f=f(T, \phi)$ according to

$$
e=f+s T
$$

As a consequence, we have

$$
s=-f_{, T}, \quad \mathrm{~d} s=\frac{1}{T}(\mathrm{~d} e-\mathrm{d} f)=\frac{1}{T} \mathrm{~d} e-\sum_{\alpha=1}^{M} \frac{1}{T} f_{, \phi_{\alpha}} \mathrm{d} \phi_{\alpha},
$$

and therefore

$$
s_{, e}=\frac{1}{T}, \quad s_{, \phi_{\alpha}}=-\frac{1}{T} f_{, \phi_{\alpha}} \quad \alpha=1, \ldots, M .
$$

We assume that the free energy density $f$ is obtained by interpolation of the individual bulk free energies $L_{\alpha} \frac{T-T_{\alpha}}{T_{\alpha}}-c_{v} T(\ln (T)-1)$ for each phase $\alpha$. Here, $L_{\alpha} \geq 0$ and $T_{\alpha}>0$ represent the latent heat and melting temperature of pure phase $\alpha$, respectively, and $c_{v}>0$ is the specific heat capacity. In the light of (2), this leads to

$$
f(T, \phi)=\sum_{\alpha=1}^{M} L_{\alpha} \frac{T-T_{\alpha}}{T_{\alpha}} \phi_{\alpha}-c_{v} T(\ln (T)-1)
$$

and we have

$$
f_{, \phi_{\alpha}}(T, \phi)=L_{\alpha} \frac{T-T_{\alpha}}{T_{\alpha}}
$$

Utilizing (4), (5), and the state equation (7), we can represent the entropy $s$ and the energy $e$ in terms of the temperature $T$ and the phase field $\phi$ according to (9)

$$
s(e, \phi)=\tilde{s}(T, \phi)=-\sum_{\alpha=1}^{M} L_{\alpha} \frac{1}{T_{\alpha}} \phi_{\alpha}+c_{v} \ln (T), \quad e=\tilde{e}(T, \phi)=-\sum_{\alpha=1}^{M} L_{\alpha} \phi_{\alpha}+c_{v} T .
$$


Though our approach could be extended to anisotropic interfacial energies [28], we choose

$$
\gamma(\phi, \nabla \phi)=|\nabla \phi|
$$

for simplicity. Finally, $\psi$ stands for the classical multi-obstacle potential $[13,5]$

$$
\psi(\phi)=\chi_{G}(\phi)+\frac{1}{2} \phi^{T} K \phi,
$$

with $\chi$ denoting the characteristic function

$$
\chi_{A}(x)=0 \text { if } x \in A \text { and } \chi_{A}(x)=\infty \text { if } x \notin A
$$

and $G$ the Gibbs simplex

$$
G=\left\{v=\left(v_{\alpha}\right) \in \mathbb{R}^{M} \mid \sum_{\alpha=1}^{M} v_{\alpha}=1 \text { and } v_{\alpha} \geq 0, \alpha=1, \ldots, M\right\} \subset \mathbb{R}^{M} .
$$

We choose the negative definite interaction matrix

$$
K=-I \in \mathbb{R}^{M, M}
$$

leading to the concave contribution $\phi^{T} K \phi=-|\phi|^{2}$ to the multi-obstacle potential.

With these specifications the entropy functional takes the form

$$
S(e, \phi)=S_{0}(e, \phi)-\chi_{\mathcal{G}}(\phi), \quad S_{0}(e, \phi)=\int_{\Omega} s(e, \phi)-\frac{\varepsilon}{2}|\nabla \phi|^{2}-\frac{1}{2 \varepsilon} \phi^{T} K \phi \mathrm{d} x
$$

where $\chi_{\mathcal{G}}$ is the characteristic functional of

$$
\mathcal{G}=\left\{v \in H^{1}(\Omega)^{M} \mid v(x) \in G \text { a.e. in } \Omega\right\} .
$$

2.2. A multi-phase Penrose-Fife system. We postulate the continuity equation

$$
e_{t}=-\nabla \cdot J_{0}(e, \phi)+q(e, \phi)
$$

with the flux

$$
J_{0}(e, \phi)=\kappa \nabla\left(\delta_{e} S\right)(e, \phi),
$$

mobility $\kappa>0$, variational derivative $\delta_{e} S$, and a source term $q(e, \phi)$ to obtain

$$
e_{t}=-\nabla \cdot \kappa \nabla\left(\delta_{e} S\right)(e, \phi)+q(e, \phi) .
$$

We assume that the outward energy flux is proportional to the difference of the temperature $T$ and a given boundary temperature $T_{\Gamma}$ or, more precisely, we prescribe

$$
J_{0} \cdot n=h_{c}\left(T-T_{\Gamma}\right)
$$

with the convection coefficient $h_{c}>0$ and the outward normal $n$ to $\Omega$. It is also convenient to introduce the inverse temperature

$$
\theta=\frac{1}{T}, \quad \theta_{\Gamma}=\frac{1}{T_{\Gamma}} .
$$

Note that for given $\phi$ the variables $e, T$, and $\theta$ can be transformed into each other due to the strictly monotone relationships (9) and (19).

In order to provide a non-decreasing entropy $S(e, \phi)$ in the course of the phase evolution, we set

$$
\varepsilon \beta \phi_{t} \in \delta_{\phi} S_{0}(e, \phi)-\partial \chi_{\mathcal{G}}(\phi),
$$

with a kinetic coefficient $\beta>0$, the variational derivative $\delta_{\phi} S_{0}(e, \phi)$, and the subdifferential $\partial \chi_{\mathcal{G}}(\phi)$ of the convex functional $\chi_{\mathcal{G}}$. For the phase field we impose homogeneous Neumann boundary conditions

$$
\frac{\partial}{\partial n} \phi_{\alpha}=0, \quad \alpha=1, \ldots, M \text {. }
$$


Utilizing (9), (10), (11), and the transformation (19), a weak formulation of the differential equations (17) and (20) with boundary conditions (18) and (21), respectively, reads as follows.

Problem 2.1 (Multi-phase Penrose-Fife system with obstacle potential). Find the phase field $\phi \in L^{2}\left(\left(0, t^{*}\right), H^{1}(\Omega)^{M}\right) \cap H^{1}\left(\left(0, t^{*}\right), L^{2}(\Omega)^{M}\right)$ and positive inverse temperature $\theta \in L^{2}\left(\left(0, t^{*}\right), H^{1}(\Omega)\right) \cap H^{1}\left(\left(0, t^{*}\right), L^{2}(\Omega)\right)$ such that

$$
\phi(\cdot, 0)=\phi^{0}, \quad \theta(\cdot, 0)=\theta^{0}
$$

holds with given initial conditions $\phi^{0} \in L^{2}(\Omega)^{M}, \theta^{0} \in L^{2}(\Omega), \theta^{0} \geq 0$ a.e. in $\Omega$ and

$$
\begin{aligned}
&\left(\varepsilon \beta \phi_{t}+\widetilde{L}-\theta L+\frac{1}{\varepsilon} K \phi, v-\phi\right)+\varepsilon(\nabla \phi, \nabla(v-\phi))+\chi_{\mathcal{G}}(v)-\chi_{\mathcal{G}}(\phi) \geq 0 \\
&\left(-L^{T} \phi_{t}+c_{v} \frac{1}{\theta^{2}} \theta_{t}-q, w\right)-(\kappa \nabla \theta, \nabla w)+\left(h_{c}\left(\frac{1}{\theta}-\frac{1}{\theta_{\Gamma}}\right), w\right)_{\Gamma}=0
\end{aligned}
$$

holds for all $v \in H^{1}(\Omega)^{M}$ and $w \in H^{1}(\Omega)$.

Here, $\left(0, t^{*}\right) \in \mathbb{R}$ is the considered time interval, $L=\left(L_{\alpha}\right)_{\alpha=1}^{M}$ and $\widetilde{L}=\left(\frac{L_{\alpha}}{T_{\alpha}}\right)_{\alpha=1}^{M}$ are constant vectors used to simplify the notation of $(6)$ and $(7)$, and $(\cdot, \cdot),(\cdot, \cdot)_{\Gamma}$ stand for the scalar product in $L^{2}(\Omega), L^{2}(\Gamma)$, respectively.

For existence and uniqueness results in the special case $M=2$, we refer to [17, Section 7.2], [37], and the references cited therein.

Proposition 2.1. The multi-phase Penrose-Fife system is thermodynamically consistent in the sense that

$$
S(e(t), \phi(t)) \geq S\left(e\left(t_{0}\right), \phi\left(t_{0}\right)\right) \quad \forall t \in\left[t_{0}, t^{*}\right] \subset\left(0, t^{*}\right]
$$

holds for any solution $(\phi, \theta)$ of Problem 2.1 with $q=0, h_{c}=0$ satisfying $\phi \in$ $C^{1}\left(\left[t_{0}, t^{*}\right], H^{1}(\Omega)^{M}\right)$ for $\left[t_{0}, t^{*}\right] \subset\left(0, t^{*}\right]$.

Proof. Since $\phi \in \mathcal{G}$ for almost all $t$ and in view of (9) and (14) we can write

$$
S(e, \phi)=\hat{S}(\theta, \phi)=\int_{\Omega}-\widetilde{L}^{T} \phi+c_{v} \ln \left(\frac{1}{\theta}\right)-\frac{\varepsilon}{2}|\nabla \phi|^{2}-\frac{1}{2 \varepsilon} \phi^{T} K \phi \mathrm{d} x .
$$

Testing (23a) with $v=\phi(t-\tau)$, dividing by $\tau>0$, and letting $\tau \rightarrow 0$ we get

$$
0 \leq \varepsilon\left\|\sqrt{\beta} \phi_{t}\right\|^{2} \leq\left(-\widetilde{L}+\theta L-\frac{1}{\varepsilon} K \phi, \phi_{t}\right)-\varepsilon\left(\nabla \phi, \nabla \phi_{t}\right)
$$

whereas testing (23b) with $w=\theta$ yields

$$
0 \leq(\kappa \nabla \theta, \nabla \theta)=\left(-L^{T} \phi_{t}+c_{v} \frac{1}{\theta^{2}} \theta_{t}, \theta\right) .
$$

Adding both we get

$$
\left.0 \leq\left(-\widetilde{L}, \phi_{t}\right)-\left(\frac{c_{v}}{\theta}, \theta_{t}\right)-\varepsilon\left(\nabla \phi, \nabla \phi_{t}\right)-\frac{1}{\varepsilon}\left(K \phi, \phi_{t}\right)=\langle\nabla \hat{S}(\theta, \phi)),\left(\theta_{t}, \phi_{t}\right)\right\rangle .
$$

Now integrating over $\left[t_{0}, t\right]$ provides the assertion.

2.3. Thin film approximation. We consider a domain of the form $\Omega=\Omega^{\prime} \times$ $(0, H) \subset \mathbb{R}^{d}, d=2,3$, with a bounded Lipschitz domain $\Omega^{\prime} \subset \mathbb{R}^{d-1}$ and $H>0$. We assume that $\Omega$ is "thin" in the sense that variations of $\phi, \phi^{0}, \theta, \theta^{0}$, and $q$ normal to $\Omega^{\prime}$ as well as the flux $J_{0}$ across $\partial \Omega^{\prime} \times(0, H)$ can be neglected and $J_{0} \cdot n(\cdot, 0)=J_{0} \cdot n(\cdot, H)$ holds a.e. in $\Omega^{\prime}$. Inserting these assumptions into (22), (23), we obtain the following thin film approximation of Problem 2.1.

Problem 2.2 (Thin film multi-phase Penrose-Fife system).

Find the phase field $\phi \in L^{2}\left(\left(0, t^{*}\right), H^{1}\left(\Omega^{\prime}\right)^{M}\right) \cap H^{1}\left(\left(0, t^{*}\right), L^{2}\left(\Omega^{\prime}\right)^{M}\right)$ and positive inverse temperature $\theta \in L^{2}\left(\left(0, t^{*}\right), H^{1}\left(\Omega^{\prime}\right)\right) \cap H^{1}\left(\left(0, t^{*}\right), L^{2}\left(\Omega^{\prime}\right)\right)$ such that

$$
\phi(\cdot, 0)=\phi^{0}, \quad \theta(\cdot, 0)=\theta^{0}
$$


holds with given initial conditions $\phi^{0} \in L^{2}(\Omega)^{M}, \theta^{0} \in L^{2}(\Omega), \theta^{0} \geq 0$ a.e. in $\Omega$ and

$$
\begin{aligned}
\left(\varepsilon \beta \phi_{t}+\widetilde{L}-\theta L+\frac{1}{\varepsilon} K \phi, v-\phi\right)+\varepsilon(\nabla \phi, \nabla(v-\phi))+\chi_{\mathcal{G}}(v)-\chi_{\mathcal{G}}(\phi) & \geq 0 \\
\left(-L^{T} \phi_{t}+c_{v} \frac{1}{\theta^{2}} \theta_{t}+h_{c}^{\prime} \frac{1}{\theta}-h_{c}^{\prime} \frac{1}{\theta_{\Gamma}}-q, w\right)-(\kappa \nabla \theta, \nabla w) & =0
\end{aligned}
$$

and $h_{c}^{\prime}=\frac{2 h_{c}}{H}$ for all $v \in H^{1}\left(\Omega^{\prime}\right)^{M}$ and $w \in H^{1}\left(\Omega^{\prime}\right)$.

Here, $(\cdot, \cdot)=(\cdot, \cdot)_{\Omega^{\prime}}$ stands for the scalar product in $L^{2}\left(\Omega^{\prime}\right)$ for ease of notation. Note that Problem 2.2 is essentially a $(d-1)$-dimensional analogue of Problem 2.1 with similar mathematical properties. For example, the thermodynamic consistency in the sense of Proposition 2.1 is still valid and all considerations concerning the discretization and algebraic solution of discretized problems to be reported below carry over from Problem 2.1 to its thin film approximation. For this reason, in the remainder we consider the more general equation

$$
\left(-L^{T} \phi_{t}+c_{v} \frac{1}{\theta^{2}} \theta_{t}+h_{\Omega} \frac{1}{\theta}-q^{\prime}, w\right)-(\kappa \nabla \theta, \nabla w)+\left(h_{\Gamma}\left(\frac{1}{\theta}-\frac{1}{\theta_{\Gamma}}\right), w\right)_{\Gamma}=0
$$

with the coefficients $h_{\Omega}, h_{\Gamma} \geq 0$, of which exactly one is zero to recover Problems 2.1 resp. 2.2. Specifically, choose $h_{\Omega}=0, h_{\Gamma}=h_{c}$, and $q^{\prime}=q$ to obtain $(23 \mathrm{~b})$ and $h_{\Omega}=h_{c}^{\prime}, h_{\Gamma}=0$, and $q^{\prime}=q+h_{c}^{\prime} \frac{1}{\theta_{\Gamma}}$ to obtain $(27 \mathrm{~b})$.

\section{Discretization}

In this section we present a discretization of Problem 2.1 and Problem 2.2 using the general equation (28) by Euler-type discretizations in time and finite elements in space. Since an efficient approximation of the phase field $\phi$ requires time-dependent, locally refined spatial grids, it is convenient to use Rothe's method [15], i.e., the variational problem (23) is first discretized in time and the resulting spatial problems are then discretized in space, independently from each other.

3.1. Implicit time discretization. In light of the well-known stiffness of the non-linear parabolic system of equations, we use a semi-implicit Euler method. More precisely, after approximating the time derivatives $\phi_{t}, \theta_{t}$ by backward finite differences with step size $\tau>0$, the nonlinearities $1 / \theta, 1 / \theta^{2}$ are approximated by first-order Taylor expansion (cf., e.g., [20, Section 6.4])

$$
\frac{1}{\theta(t)} \doteq \frac{2}{\theta(t-\tau)}-\frac{\theta(t)}{(\theta(t-\tau))^{2}}, \quad \frac{1}{\theta(t)^{2}} \doteq \frac{3}{\theta(t-\tau)^{2}}-\frac{2 \theta(t)}{\theta(t-\tau)^{3}} .
$$

In particular, this leads to

$$
\frac{1}{\theta(t)^{2}} \theta_{t}(t) \doteq \frac{1}{\theta(t)^{2}} \frac{\theta(t)-\theta(t-\tau)}{\tau}=\frac{1}{\tau}\left(\frac{1}{\theta(t)}-\frac{\theta(t-\tau)}{\theta(t)^{2}}\right) \doteq \frac{1}{\tau}\left(\frac{\theta(t)}{\theta(t-\tau)^{2}}-\frac{1}{\theta(t-\tau)}\right) .
$$

Only the concave term $\frac{1}{\varepsilon} K \phi$ is taken explicitly [19, 28], trading unconditional stability for a potential loss of accuracy, c.f. [11] or [6, section 6.3.1].

For simplicity, we utilize the uniform time step size $\tau=t^{*} / n^{*}$ with given $n^{*} \in \mathbb{N}$, and denote the approximations of $\phi\left(t_{n}\right), \theta\left(t_{n}\right)$ at $t_{n}=n \tau, n=1, \ldots, n^{*}$ by $\phi^{n}, \theta^{n}$, respectively. The spatial problem to be solved in the $n$-th time step then reads as follows.

Problem 3.1 (Spatial multi-phase Penrose-Fife system with obstacle potential). Find the phase field $\phi^{n} \in H^{1}(\Omega)^{M}$ and positive inverse temperature $\theta^{n} \in H^{1}(\Omega)$ such that

$$
\begin{aligned}
a\left(\phi^{n}, v-\phi^{n}\right)+\chi_{\mathcal{G}}(v)-\chi_{\mathcal{G}}\left(\phi^{n}\right)+b\left(v-\phi^{n}, \theta^{n}\right) & \geq \ell_{1}^{n}\left(v-\phi^{n}\right) \\
b\left(\phi^{n}, w\right)-c^{n}\left(\theta^{n}, w\right) & =\ell_{2}^{n}(w)
\end{aligned}
$$


holds with the bilinear forms

$$
\begin{aligned}
a\left(v, v^{\prime}\right) & =\varepsilon\left(\beta v, v^{\prime}\right)+\varepsilon \tau\left(\nabla v, \nabla v^{\prime}\right), \\
b(v, w) & =-\tau\left(L^{T} v, w\right), \\
c^{n}\left(w, w^{\prime}\right) & =\tau\left(\frac{c_{v}+\tau h_{\Omega}}{\left(\theta^{n-1}\right)^{2}} w, w^{\prime}\right)+\tau^{2}\left(\frac{h_{\Gamma}}{\left(\theta^{n-1}\right)^{2}} w, w^{\prime}\right)_{\Gamma}+\tau^{2}\left(\kappa \nabla w, \nabla w^{\prime}\right)
\end{aligned}
$$

and the linear functionals

$$
\begin{aligned}
& \ell_{1}^{n}(v)=\left(\varepsilon \beta \phi^{n-1}-\tau \widetilde{L}-\frac{\tau}{\varepsilon} K \phi^{n-1}, v\right), \\
& \ell_{2}^{n}(w)=\left(\tau^{2} q^{\prime}-\tau L^{T} \phi^{n-1}-\tau \frac{c_{v}+2 \tau h_{\Omega}}{\theta^{n-1}}, w\right)-\tau^{2} h_{\Gamma}\left(\frac{2}{\theta^{n-1}}-\frac{1}{\theta_{\Gamma}}, w\right)_{\Gamma}
\end{aligned}
$$

defined for all $v, v^{\prime} \in H^{1}(\Omega)^{M}$ and $w, w^{\prime} \in H^{1}(\Omega)$.

Proposition 3.1. The time-discrete multi-phase Penrose-Fife system is thermodynamically consistent in the sense that

$$
S\left(e^{n}, \phi^{n}\right) \geq S\left(e^{n-1}, \phi^{n-1}\right)
$$

holds for any solution $\left(\phi^{n}, \theta^{n}\right)$ of Problem 3.1 with $q=0, h_{c}=0$. Here, $e^{n}=$ $\tilde{e}\left(\frac{1}{\theta^{n}}, \phi^{n}\right)$ is defined according to $(9)$.

Proof. Testing equation (29a) with $v=\phi^{n-1}$ yields

$$
\frac{\varepsilon}{2}\left|\nabla \phi^{n}\right|^{2}-\frac{\varepsilon}{2}\left|\nabla \phi^{n-1}\right|^{2} \leq\left(\widetilde{L}-L \theta^{n}+\frac{1}{\varepsilon} K \phi^{n-1}, \phi^{n-1}-\phi^{n}\right)
$$

and testing equation (29b) with $w=\theta^{n}$ for $q=0, h_{c}=0$ yields

$$
0 \leq \tau \kappa\left|\nabla \theta^{n}\right|^{2}=\left(L \theta^{n}, \phi^{n-1}-\phi^{n}\right)+c_{v} \int_{\Omega} \frac{\theta^{n}}{\theta^{n-1}}\left(1-\frac{\theta^{n}}{\theta^{n-1}}\right) \mathrm{d} x .
$$

Adding both inequalities, we obtain

$$
\begin{aligned}
& -\left(\widetilde{L}, \phi^{n-1}-\phi^{n}\right)-\frac{\varepsilon}{2}\left(\left|\nabla \phi^{n-1}\right|^{2}-\left|\nabla \phi^{n}\right|^{2}\right) \\
& \quad \leq c_{v} \int_{\Omega} \frac{\theta^{n}}{\theta^{n-1}}\left(1-\frac{\theta^{n}}{\theta^{n-1}}\right) \mathrm{d} x+\frac{1}{\varepsilon}\left(K \phi^{n-1}, \phi^{n-1}-\phi^{n}\right)
\end{aligned}
$$

which, using the representation (25), finally can be used to show

$$
S\left(e^{n-1}, \phi^{n-1}\right)-S\left(e^{n}, \phi^{n}\right) \leq \int_{\Omega} c_{v}\left(\ln \left(\frac{\theta^{n}}{\theta^{n-1}}\right)+\frac{\theta^{n}}{\theta^{n-1}}\left(1-\frac{\theta^{n}}{\theta^{n-1}}\right)\right) \mathrm{d} x .
$$

Since the right hand side is non-positive, this provides the assertion.

It turns out that the system (29) can be regarded as optimality conditions for a Lagrange-functional.

Proposition 3.2. Problem 3.1 is equivalent to find $\phi^{n} \in H^{1}(\Omega)^{M}$ and $\theta^{n} \in H^{1}(\Omega)$ such that

$$
\mathcal{L}^{n}\left(\phi^{n}, w\right) \leq \mathcal{L}^{n}\left(\phi^{n}, \theta^{n}\right) \leq \mathcal{L}^{n}\left(v, \theta^{n}\right) \quad \forall v \in H^{1}(\Omega)^{M}, w \in H^{1}(\Omega),
$$

with the Lagrangian $\mathcal{L}^{n}$ given by

$$
\mathcal{L}^{n}(v, w)=\mathcal{J}^{n}(v)-\ell_{1}^{n}(v)+b(v, w)-\ell_{2}^{n}(w)-\frac{1}{2} c^{n}(w, w)
$$

denoting $\mathcal{J}^{n}(v)=\frac{1}{2} a(v, v)+\chi_{\mathcal{G}}(v)$.

Theorem 3.1. Let $\phi^{n-1} \in \mathcal{G}, \theta^{n-1} \in H^{1}(\Omega)$ and $\theta^{n-1} \geq c$ a.e. in $\Omega$ with a positive constant $c$. Then the spatial Problem 3.1 admits a unique solution. 
Proof. Under the given assumptions, $\ell_{1}^{n}$ and $\ell_{2}^{n}$ are bounded linear functionals and $a(\cdot, \cdot)$ is symmetric and coercive. Since $\mathcal{G}$ is closed and convex the functional $\mathcal{L}^{n}(\cdot, w)$ is strictly convex, coercive, and lower semi-continuous for all fixed $w \in H^{1}(\Omega)$. Thus, we can define the dual functional

$$
h(w)=-\inf _{v \in H^{1}(\Omega)^{M}} \mathcal{L}^{n}(v, w)=\left(\mathcal{J}^{n}\right)^{*}\left(\ell_{1}^{n}-b(\cdot, w)\right)+\frac{1}{2} c^{n}(w, w)+\ell_{2}^{n}(w) .
$$

Here, $\left(\mathcal{J}^{n}\right)^{*}$ is the convex and continuous polar of $\mathcal{J}^{n}$. Notice that $h$ is also convex and continuous because $c^{n}(\cdot, \cdot)$ is symmetric and positive-definite.

By integrability of $\left(\theta^{n-1}\right)^{2}$ there must be a subset $\Omega^{\prime} \subset \Omega$ with positive measure and a constant $c_{2}>0$ such that $\theta^{n-1} \leq c_{2}$ on $\Omega^{\prime}$. As a consequence we get

$$
c^{n}(w, w) \geq \frac{c_{v} \tau}{c_{2}^{2}}\|w\|_{L^{2}\left(\Omega^{\prime}\right)}^{2}+\tau^{2} \kappa\|\nabla w\|^{2}
$$

and therefore coercivity of $c^{n}(\cdot, \cdot)$. Hence, $h$ is coercive, continuous, and strictly convex and thus has a unique minimizer. Existence and uniqueness now follows from the fact that $\left(\phi^{n}, \theta^{n}\right)$ is a solution of Problem 3.1, if and only if $\theta^{n}$ is a minimizer of $h$ and $\phi^{n}=\arg \min _{v \in H^{1}(\Omega)^{M}} \mathcal{L}^{n}\left(v, \theta^{n}\right)$.

In general it is not clear if the uniform positivity of the inverse temperature is preserved by solving Problem 3.1. We refer, however, to [37] for such kind of results in the scalar case $M=2$.

3.2. Adaptive finite element discretization. We will now consider the adaptive finite element discretization of the spatial Problem 3.1 for an individual fixed time stepIn order to improve readability we will from now on drop all superscripts $(\cdot)^{n}$ that identify the current time step. We will designate quantities from the previous time step by the superscript $(\cdot)^{\text {old }}$ whenever necessary.

3.2.1. Finite element discretization. In the following, we assume that $\mathcal{T}$ is a simplicial grid that is either conforming or obtained via local hanging node refinement of a conforming initial grid. We will discretize the spatial Problem 3.1 with respect to the conforming first order finite element space

$$
\mathcal{S}=\mathcal{S}(\mathcal{T})=\left\{v \in C(\bar{\Omega})|v|_{\tau} \text { is affine } \forall \tau \in \mathcal{T}\right\} \subset H^{1}(\Omega)
$$

Notice that $\mathcal{S}$ has a uniquely defined nodal basis $\left\{\lambda_{p} \mid p \in \mathcal{N}\right\}$ satisfying $\lambda_{p}(q)=\delta_{p q}$ for all $p, q \in \mathcal{N}$ where $\mathcal{N}$ is the set of non-hanging nodes of $\mathcal{T}$. If the grid is obtained by uniform or local hanging node refinement, the non-conforming mesh hierarchy induces a natural hierarchy of subspaces of $\mathcal{S}$ that can be used in geometric multigrid methods. For a detailed discussion of finite element spaces on hierarchies of non-conforming, locally refined grids we refer to $[25,30]$.

Note that linearity implies that the Gibbs constraint can be evaluated node-wise, i.e.,

$$
\mathcal{G} \cap \mathcal{S}^{M}=\left\{v \in \mathcal{S}^{M} \mid v(p) \in G \forall p \in \mathcal{N}\right\} .
$$

Problem 3.2 (Discrete spatial multi-phase Penrose-Fife system). Find the phase field $\phi_{\mathcal{T}} \in \mathcal{S}^{M}$ and positive inverse temperature $\theta_{\mathcal{T}} \in \mathcal{S}$ such that

$$
\begin{aligned}
a\left(\phi_{\mathcal{T}}, v-\phi_{\mathcal{T}}\right)+\chi_{\mathcal{G}}(v)-\chi_{\mathcal{G}}\left(\phi_{\mathcal{T}}\right)+b\left(v-\phi_{\mathcal{T}}, \theta_{\mathcal{T}}\right) & \geq \ell_{1}\left(v-\phi_{\mathcal{T}}\right), \\
b\left(\phi_{\mathcal{T}}, w\right)-c\left(\theta_{\mathcal{T}}, w\right) & =\ell_{2}(w)
\end{aligned}
$$

holds for all $v \in \mathcal{S}^{M}$ and $w \in \mathcal{S}$.

Here, $\ell_{1}, \ell_{2}$, and $c(\cdot, \cdot)$ are defined as in Problem 3.1 but with $\phi^{n-1}$ and $\theta^{n-1}$ replaced by their finite element approximations $\phi^{\text {old }}$ and $\theta^{\text {old }}$. To avoid negative values for $\theta_{\mathcal{T}}$ due to overshooting when $q^{\prime}$ is not resolved by the grid, we replace the latter by its interpolation in $\mathcal{S}$. Notice that $\phi^{\text {old }}$ and $\theta^{\text {old }}$ are finite element 
functions on a grid $\mathcal{T}^{\text {old }}$. In case of adaptive refinement, $\mathcal{T}^{\text {old }}$ is usually different from $\mathcal{T}$. See [30] for a detailed discussion.

In analogy to its continuous counterpart, Problem 3.2 can be written as a nonlinear, non-smooth saddle point problem.

Proposition 3.3. Problem 3.2 is equivalent to finding $\phi_{\mathcal{T}} \in \mathcal{S}^{M}$ and $\theta_{\mathcal{T}} \in \mathcal{S}$ such that

$$
\mathcal{L}\left(\phi_{\mathcal{T}}, w\right) \leq \mathcal{L}\left(\phi_{\mathcal{T}}, \theta_{\mathcal{T}}\right) \leq \mathcal{L}\left(v, \theta_{\mathcal{T}}\right) \quad \forall v \in \mathcal{S}^{M}, w \in \mathcal{S}
$$

with the Lagrangian $\mathcal{L}$ given according to $(33)$.

Existence and uniqueness are also inherited from the continuous case.

Theorem 3.2. Let $\phi^{\text {old }} \in \mathcal{G}, \theta^{\text {old }} \in H^{1}(\Omega)$ and $\theta^{\text {old }} \geq c$ a.e. in $\Omega$ with a positive constant $c$. Then the spatial Problem 3.2 admits a unique solution.

Proof. The proof can be carried out using the same arguments as in the proof of Theorem 3.1.

3.2.2. Hierarchical a posteriori error estimation. As the phase field $\phi$ is expected to strongly vary across the phase boundaries, spatial adaptivity based on a posteriori error estimates is mandatory. Similarly, the consumption of heat by phase changes may lead to strongly varying $\theta$. Hierarchical error estimates rely on the solution of local defect problems. While originally introduced for linear elliptic problems $[16,21,34,49]$ this technique was successfully extended to non-linear problems [4], constrained minimization $[35,38,40,46,50]$ and non-smooth saddle point problems $[27,30,25]$.

Following [25, 27, 30], we now derive an a posteriori error estimate by a suitable approximation of the defect problem associated with the defect Lagrangian

$$
\mathcal{D}\left(e_{\phi}, e_{\theta}\right)=\mathcal{L}\left(\phi_{\mathcal{T}}+e_{\phi}, \theta_{\mathcal{T}}+e_{\theta}\right) .
$$

In the first step the defect problem is discretized with respect to a larger finite element space $\mathcal{Q}^{M} \times \mathcal{Q}$, where $\mathcal{Q}=\mathcal{S}\left(\mathcal{T}^{\prime}\right)$ is defined analogously to (34) for the grid $\mathcal{T}^{\prime}$ obtained by uniform refinement of $\mathcal{T}$. Note that we have $\mathcal{Q}=\mathcal{S} \oplus \mathcal{V}$ with $\mathcal{V}$ denoting the incremental space

$$
\mathcal{V}=\operatorname{span}\left\{\lambda_{p}^{\prime} \mid p \in \mathcal{E}\right\} \subset \operatorname{span}\left\{\lambda_{p}^{\prime} \mid p \in \mathcal{N}^{\prime}\right\}=\mathcal{S}^{\prime} .
$$

Here, $\mathcal{N}^{\prime}$ denotes the set of non-hanging nodes in $\mathcal{T}^{\prime},\left\{\lambda_{p}^{\prime} \mid p \in \mathcal{N}^{\prime}\right\}$ the nodal basis of $\mathcal{S}^{\prime}$, and $\mathcal{E}=\mathcal{N}^{\prime} \backslash \mathcal{N}$ is the set of all edge mid points in $\mathcal{T}$ that are non-hanging in $\mathcal{T}^{\prime}$.

In the second step, the discrete defect problem is localized by ignoring the coupling between $\mathcal{S}$ and $\mathcal{V}$ and also the coupling between $\lambda_{p}^{\prime}$ for all $p \in \mathcal{E}$. Denoting $\mathcal{D}_{p}(r, s)=\mathcal{D}\left(r \lambda_{p}^{\prime}, s \lambda_{p}^{\prime}\right)$, this results in the local saddle point problems

$\left(e_{\phi, p}, e_{\theta, p}\right) \in \mathbb{R}^{M} \times \mathbb{R}: \quad \mathcal{D}_{p}\left(e_{\phi, p}, s\right) \leq \mathcal{D}_{p}\left(e_{\phi, p}, e_{\theta, p}\right) \leq \mathcal{D}_{p}\left(r, e_{\theta, p}\right) \quad \forall(r, s) \in \mathbb{R}^{M} \times \mathbb{R}$ for all $p \in \mathcal{E}$ that give rise to the hierarchical a posteriori error estimate

$$
\eta=\left(\sum_{p \in \mathcal{E}} \eta_{p}^{2}\right)^{\frac{1}{2}}, \quad \eta_{p}^{2}=\left\|e_{\phi, p} \lambda_{p}^{\prime}\right\|_{\phi}^{2}+\left\|e_{\theta, p} \lambda_{p}^{\prime}\right\|_{\theta}^{2}, \quad p \in \mathcal{E}
$$

with the problem-dependent norms

$$
\|v\|_{\phi}^{2}=a(v, v), \quad\|w\|_{\theta}^{2}=c(w, w)
$$

on $\mathcal{V}^{M}, \mathcal{V}$, respectively. 
3.2.3. Adaptive mesh refinement. The initial grid for the adaptive refinement should be sufficiently fine to detect basic features of the unknown spatial approximation and sufficiently coarse for efficiency of the overall adaptive procedure. The construction of such a grid starts with the grid $\mathcal{T}^{\text {old }}$ from the preceding time step. In the first time step, we select a suitable, uniformly refined grid $\mathcal{T}^{\text {old }}$.

We begin by coarsening $\mathcal{T}^{\text {old }}$. To this end, we keep all simplices from the grid $\mathcal{T}^{\text {old }}$ from the preceding time step that were obtained by at most $j_{\text {min }}$ refinements. In addition, we keep all simplices $\tau$ such that $\phi^{\text {old }}$ exhibits a strong local variation on $\tau$ that is not visible after coarsening, i.e., such that

$$
\left\|\left|\nabla\left(I_{\tau} \phi^{\text {old }}\right)\right|\right\|_{L^{\infty}(\tau)} \geq \operatorname{Tol}_{\text {derefine }} \quad \text { and } \quad\left\|\left|\nabla\left(I_{\tau^{\prime}} \phi^{\text {old }}\right)\right|\right\|_{L^{\infty}\left(\tau^{\prime}\right)}<\operatorname{Tol}_{\text {derefine }}
$$

holds with $\tau^{\prime}$ denoting the simplex resulting from coarsening of $\tau$. $I_{\tau}$ and $I_{\tau^{\prime}}$ are the linear interpolation operators to $\tau$ and $\tau^{\prime}$, respectively. This set of simplices is completed by additional local refinements. Possible additional refinement is used to uniformly bound the ratio of diameters of adjacent simplices.

The adaptive mesh refinement of the resulting initial grid $\mathcal{T}$ is based on the local error indicators $\eta_{p}$ defined in (38). In each step, the indicators $\eta_{p_{i}}, i=1, \ldots,|\mathcal{E}|$, are arranged with decreasing value, to determine the minimal number $i_{0}$ of indicators such that

$$
\sum_{i=1}^{i_{0}} \eta_{p_{i}}^{2} \geqslant \rho \eta^{2}
$$

holds with a given parameter $\rho \in[0,1]$. Then all simplices $\tau \in \mathcal{T}$ with the property $p_{i} \in \tau$ for some $p_{i}$ with $i \leq i_{0}$ are marked for refinement [22]. Each marked simplex is partitioned by (red) refinement [9, 14]. Again, possible additional refinement is used to uniformly bound the ratio of diameters of adjacent simplices. The refinement process is stopped, when the estimated relative error is less than a given tolerance $\mathrm{Tol}_{\text {adapt }}>0$, i.e., if

$$
\eta<\operatorname{Tol}_{\text {adapt }} \cdot\left(\left\|\phi_{\mathcal{T}}\right\|_{\phi}^{2}+\left\|\theta_{\mathcal{T}}\right\|_{\theta}^{2}\right)^{\frac{1}{2}}
$$

\section{Algebraic solution}

Several methods have been proposed for the algebraic solution of discretized multi-phase field equations. The solution of multi-phase Allen-Cahn-type equations via primal-dual active set methods was discussed in [10] and multigrid methods for such problems where proposed in $[33,39]$. In contrast to the second order problems with minimization structure considered there, Problem 3.2 is a saddle point problem for a discretized fourth order equation. For similar problems resulting from multi-component Cahn-Hilliard systems block Gauß-Seidel-type algorithms with component-wise and vertex-wise blocking where proposed in [12] and [43], respectively. To overcome the mesh-dependence of the Gauß-Seidel approach, a nonsmooth Schur-Newton method was proposed in [29].

While, for Cahn-Hilliard-type systems, the local sum constraint in $G$ can be enforced using the chemical potential as a natural Lagrange multiplier (cf. [29]), Problem 3.2 is structurally different and the introduction of such a multiplier would change the structure of the problem. Therefore, we now introduce a nonsmooth Schur-Newton method that does not involve such a multiplier.

4.1. Matrix notation. For the presentation of the algebraic solver for the iterative solution of Problem 3.2 we first formulate this problem in terms of coefficient vectors and matrices. To this end let $N=\operatorname{dim} \mathcal{S}$ and introduce an enumeration of the nodes $\mathcal{N}=\left\{p_{1}, \ldots, p_{N}\right\}$. To simplify the presentation, we use the abbreviated 
notation $\lambda_{k}=\lambda_{p_{k}}$ for the nodal basis $\lambda_{1}, \ldots, \lambda_{N}$ of $\mathcal{S}$ and introduce the basis $\lambda^{1}, \ldots, \lambda^{M N}$ of $\mathcal{S}^{M}$ where $\lambda^{\pi(i, k)}=b^{i} \lambda_{k}, b^{i} \in \mathbb{R}^{M}$ is the $i$-th Euclidean basis vector, and $\pi:\{1, \ldots, N\} \times\{1, \ldots, M\}$ is the bijective index map given by

$$
\pi(k, i)=i+M(k-1) .
$$

For $v \in \mathcal{S}^{M}, w \in \mathcal{S}$ we then get the associated coefficient vectors $V \in \mathbb{R}^{M N}, W \in$ $\mathbb{R}^{N}$

$$
v=\sum_{i=1}^{M N} V_{i} \lambda^{i}, \quad w=\sum_{i=1}^{N} W_{i} \lambda_{i} .
$$

Using the matrices $A \in \mathbb{R}^{M N, M N}, B \in \mathbb{R}^{N, M N}, C \in \mathbb{R}^{N, N}$ and vectors $F \in$ $\mathbb{R}^{M N}, G \in \mathbb{R}^{N}$ given by

$$
A_{i j}=a\left(\lambda^{j}, \lambda^{i}\right), \quad B_{i j}=b\left(\lambda^{j}, \lambda_{i}\right), \quad C_{i j}=c\left(\lambda_{j}, \lambda_{i}\right), \quad F_{i}=\ell_{1}\left(\lambda^{i}\right), \quad G_{i}=\ell_{2}\left(\lambda_{i}\right),
$$

and the characteristic functional $\chi_{G_{N}}: \mathbb{R}^{M N} \rightarrow \mathbb{R} \cup\{\infty\}$ of

$$
G_{N}=\left\{V \in \mathbb{R}^{M N} \mid\left(V_{\pi(k, i)}\right)_{i=1, \ldots, M} \in G \quad \forall k\right\}=\left\{V \in \mathbb{R}^{M N} \mid \sum_{i=1}^{M N} V_{i} \lambda^{i} \in \mathcal{G}\right\}
$$

Problem 3.2 can be written as:

Problem 4.1 (Algebraic variational inequality).

Find the coefficient vectors $\Phi \in \mathbb{R}^{M N}$ and $\Theta \in \mathbb{R}^{N}$ of $\phi_{\mathcal{T}}$ and $\theta_{\mathcal{T}}$, respectively, such that

$$
\begin{aligned}
\langle A \Phi, V-\Phi\rangle+\chi_{G_{N}}(V)-\chi_{G_{N}}(\Phi)+\left\langle B^{T} \Theta, V-\Phi\right\rangle & \geq\langle F, V-\Phi\rangle, \\
B \Phi-C \Theta & =G
\end{aligned}
$$

holds for all $V \in \mathbb{R}^{M N}$.

Problem 4.1 can equivalently be written in operator notation as a non-linear saddle point problem using in turn the subdifferential of $\chi_{G_{N}}$.

Problem 4.2 (Discrete saddle point problem).

Find the coefficient vectors $\Phi \in \mathbb{R}^{M N}$ and $\Theta \in \mathbb{R}^{N}$ of $\phi_{\mathcal{T}}$ and $\theta_{\mathcal{T}}$, respectively, such that

$$
\left(\begin{array}{cc}
A+\partial \chi_{G_{N}} & B^{T} \\
B & -C
\end{array}\right)\left(\begin{array}{c}
\Phi \\
\Theta
\end{array}\right) \ni\left(\begin{array}{l}
F \\
G
\end{array}\right) .
$$

For later reference we note that the Lagrangian for this saddle point problem is given by the following discrete analogue

$$
L(V, W)=J(V)-\langle F, V\rangle+\langle B V-G, W\rangle-\frac{1}{2}\langle C W, W\rangle
$$

of $\mathcal{L}^{n}$ where $J(V)=\frac{1}{2}\langle A V, V\rangle+\chi_{G_{N}}(V)$ is the analogue of $\mathcal{J}^{n}$.

4.2. Non-smooth Schur-Newton multigrid methods. In the context of nonsmooth Schur-Newton methods as introduced in [25, 29], it is shown that problems of the form of Problem 4.2 can equivalently be formulated as the following dual minimization problem.

Problem 4.3 (Dual minimization problem).

Find $\Theta \in \mathbb{R}^{N}$ such that

$$
h(\Theta) \leq h(W) \quad \forall W \in \mathbb{R}^{N}
$$

where $h: \mathbb{R}^{N} \rightarrow \mathbb{R}$ is the dual functional

$$
h(W)=-\inf _{V \in \mathbb{R}^{M N}} L(V, W)=-L(\Phi(W), W)
$$

and $\Phi(W)=\left(A+\partial \chi_{G_{N}}\right)^{-1}\left(F-B^{T} W\right)$. 
Proposition 4.1. Problems 4.2 and 4.3 are equivalent in the sense that $(\Phi, \Theta)$ solves Problem 4.2 if and only if $\Theta$ solves Problem 4.3 and $\Phi=\Phi(\Theta)$. Furthermore, the dual functional $h: \mathbb{R}^{N} \rightarrow \mathbb{R}$ is convex and continuously differentiable with the Lipschitz-continuous derivative

$$
\begin{aligned}
\nabla h(W) & =-B \Phi(W)+C W+G \\
& =-B\left(A+\partial \chi_{G_{N}}\right)^{-1}\left(F-B^{T} W\right)+C W+G .
\end{aligned}
$$

The proof of Proposition 4.1 can be done analogously to the proof of [26, Theorem 2.1]. This proof also shows that $h$ can be written as

$$
h(W)=J^{*}\left(F-B^{T} W\right)+\frac{1}{2}\langle C W, W\rangle+\langle G, W\rangle
$$

where $J^{*}: \mathbb{R}^{M N} \rightarrow \mathbb{R}$ is the polar (or conjugate) functional of $J$ which is convex itself. This especially shows that $h$ is a strongly convex functional because $C$ is positive definite due to coercivity of the associated bilinear form $c(\cdot, \cdot)$ (cf. proof of Theorem 3.1).

As a consequence of Proposition 4.1 we can apply gradient-related descent methods of the form

$$
\Theta^{\nu+1}=\Theta^{\nu}+\rho_{\nu} D^{\nu}
$$

where $D^{\nu} \in \mathbb{R}^{N}$ is a decent direction and $\rho_{\nu}$ a step size. The non-smooth SchurNewton method as introduced in $[26,25,29]$ is such a descent method where $D^{\nu}$ is taken to be

$$
D^{\nu}=-S_{\nu}^{-1} \nabla h\left(\Theta^{\nu}\right)
$$

and $S_{\nu} \in \mathbb{R}^{N, N}$ is a generalized linearization of the non-smooth, non-linear but Lipschitz continuous Schur complement operator $-\nabla h$ at $\Theta^{\nu}$.

The derivation of $S_{\nu}$ essentially amounts to deriving a generalized linearization of the operator $\left(A+\partial \chi_{G_{N}}\right)^{-1}$ at $Y=F-B^{T} \Theta^{\nu}$. For simple component-wise bound constraints is has been shown in [26] that such a linearization is given by $\left(A_{\mathcal{W}(X)}\right)^{+}$. Here, $A_{\mathcal{W}(X)}$ is the restriction of $A$ to $\mathcal{W}(X) \times \mathcal{W}(X), \mathcal{W}(X)$ is the maximal subspace such that $J$ is locally smooth in

$$
(X+\mathcal{W}(X)) \cap U_{X}
$$

for some neighborhood $U_{X}$ of $X=\left(A+\partial \chi_{G_{N}}\right)^{-1}(Y)$, and $(\cdot)^{+}$is the Moore-Penrose pseudoinverse or, equivalently, the inverse of $A_{\mathcal{W}(X)}: \mathcal{W}(X) \rightarrow \mathcal{W}(X)$.

For the simplex constraints in the present problem we will use exactly the same approach. In the following we will outline the construction of $A_{\mathcal{W}(X)}$ for local simplex constraints following [33]. To this end we identify vectors $V \in \mathbb{R}^{M N}$ with block-structured vectors $\hat{V} \in\left(\mathbb{R}^{M}\right)^{N}$ such that $V_{\pi(k, i)}=\left(\hat{V}_{k}\right)_{i}$.

Due to the product structure

$$
G_{N}=\left\{V \in \mathbb{R}^{M N} \mid \hat{V} \in G^{N}\right\},
$$

of the feasible set $G_{N}$ we can determine the subspace $\mathcal{W}(X)$ in each block individually. Hence $\mathcal{W}(X)$ takes the form

$$
\mathcal{W}(X)=\left\{V \in \mathbb{R}^{M N} \mid \hat{V} \in \prod_{k=1}^{N} \mathbb{W}\left(\hat{X}_{k}\right)\right\}
$$

where $\mathbb{W}\left(\hat{X}_{k}\right)$ is the maximal subspace where $\chi_{G}$ is locally smooth near $\hat{X}_{k}$. As outlined in $[33]$ the local subspace $\mathbb{W}(\xi)$ is given by

$$
\mathbb{W}(\xi)=\operatorname{span}\left\{b^{i}-b^{j} \in \mathbb{R}^{M} \mid 1 \leq i<j \leq M, \xi_{i}>0, \xi_{j}>0\right\}
$$


for $\xi \in \mathbb{R}^{M}$. Since $\mathcal{W}(X)$ is a product space the orthogonal projection $\mathcal{P}_{\mathcal{W}(X)}$ : $\mathbb{R}^{M N} \rightarrow \mathcal{W}(X)$ is given by a block diagonal matrix where the $k$-th diagonal block is the orthogonal projection $\mathcal{P}_{\mathbb{W}\left(\hat{X}_{k}\right)}: \mathbb{R}^{M} \rightarrow \mathbb{W}\left(\hat{X}_{k}\right)$. For an explicit representation of $\mathcal{P}_{\mathbb{W}\left(\hat{X}_{k}\right)} \in \mathbb{R}^{M, M}$ we refer to [33]. Using $\mathcal{P}_{\mathcal{W}(X)}$ we now get

$$
A_{\mathcal{W}(X)}=\mathcal{P}_{\mathcal{W}(X)} A \mathcal{P}_{\mathcal{W}(X)}
$$

Although the chain rule does in general not hold true for generalized Jacobians in the sense of Clarke (see, e.g. [25]), we define a generalized linearization of the non-linear Schur complement operator $-\nabla h$ at $\Theta^{\nu}$ in an analogous manner by

$$
S_{\nu}=B\left(A_{\mathcal{W}\left(\Phi\left(\Theta^{\nu}\right)\right)}\right)^{+} B^{T}+C .
$$

As a consequence of the convexity of $h$ we can show global convergence.

Theorem 4.1. Assume that the step sizes $\rho_{\nu}$ are efficient (cf. [44, 26]), then the iterates produced by the descent method (44) with Schur-Newton directions (45) for $S_{\nu}$ given by (46) converge to the solution $\Theta$ of Problem 4.3 for any initial guess $\Theta^{0} \in \mathbb{R}^{N}$.

Proof. Notice that the $S_{\nu}$ are uniformly bounded from above and below. Hence global convergence follows from [26, Theorem 4.2].

Efficient step sizes $\rho_{\nu}$ as required in Theorem 4.1 can be obtained by classical step size rules like, e.g., the Armijo rule or bisection. Notice that it is not necessary to evaluate $S_{\nu}^{-1}$ exactly in (45) because global convergence is preserved as long as the approximation of $S_{\nu}^{-1}$ is sufficiently accurate. Since the dual functional $h$ is strongly convex, one can also show global linear convergence with a rate depending on the bounds for $S_{\nu}$ and the step size rule. For further details we refer to [26].

During each iteration of the algorithm two types of subproblems have to be solved. The evaluation of $-\nabla h\left(\Theta^{\nu}\right)$ requires to compute $\Phi\left(\Theta^{\nu}\right)=\left(A+\partial \chi_{G_{N}}\right)^{-1}\left(F-B^{T} \Theta^{\nu}\right)$. This is equivalent to minimizing $J(\cdot)+\left\langle B^{T} \Theta^{\nu}, \cdot\right\rangle$, i.e., a convex minimization problem for a quadratic functional with local simplex constraints. If the step size rule requires several trial steps further problems of this type have to be solved for each evaluation of $h$ or $\nabla h$. These convex minimization problems can efficiently be solved using non-linear multigrid methods [39, 33]. More precisely the TNNMG method for simplex-constrained problems as proposed in [33] allows to solve these problems with an effective complexity of $O\left(M^{2} N\right)$. This method was used in all numerical examples presented below.

The second type of subproblems are the linear problems (45) for the symmetric positive definite operators $S_{\nu}$. Since each $S_{\nu}$ is a linear Schur complement this is equivalent to solving the linear saddle point problem

$$
\left(\begin{array}{cc}
A_{\mathcal{W}\left(\Phi\left(\Theta^{\nu}\right)\right)} & \left(B \mathcal{P}_{\mathcal{W}\left(\Phi\left(\Theta^{\nu}\right)\right)}\right)^{T} \\
B \mathcal{P}_{\mathcal{W}\left(\Phi\left(\Theta^{\nu}\right)\right)} & -C
\end{array}\right)\left(\begin{array}{c}
\tilde{V}^{\nu} \\
D^{\nu}
\end{array}\right)=\left(\begin{array}{c}
0 \\
\nabla h\left(\Theta^{\nu}\right)
\end{array}\right)
$$

whose solution is unique in $\left(\operatorname{ker} \mathcal{P}_{\mathcal{W}\left(\Phi\left(\Theta^{\nu}\right)\right)}\right)^{\perp} \times \mathbb{R}^{N}$. In the numerical examples presented below we used a linear multigrid method with a Vanka-type smoother to solve these problems. Notice that there is no convergence proof for this linear iterative method. To increase its robustness it can be used as preconditioner for a GMRes iteration.

\section{NUMERICAL EXPERIMENTS}

All our computations are based on a non-dimensionalized version of the PenroseFife systems stated in Problem 2.1 and Problem 2.2, respectively, as obtained by 
setting

$$
\theta=\frac{T_{\mathrm{ref}}}{T}
$$

instead of (19). While the order parameter $\phi_{1}$ is representing the liquid fraction, the order parameters $\phi_{2}, \ldots, \phi_{M}$ are associated with certain solid states, as, e.g., crystalline structures, of the given material. With this in mind, the positive reference value $T_{\text {ref }} \in \mathbb{R}$ is chosen to be the melting temperature and we set $T_{\text {ref }}=T_{1}=\cdots=T_{M}=1$. Accordingly we set $L_{1}=0$ and $L_{2}=\cdots=L_{M}>0$ in all our computational examples.

Efficient step sizes $\rho_{\nu}$ for the Schur-Newton iteration (44) as required in Theorem 4.1 are determined by bisection. The iteration is stopped, once the criterion

$$
\frac{\left\|\theta^{\nu+1}-\theta^{\nu}\right\|_{\theta}}{\left\|\theta^{\nu}\right\|_{\theta}} \leq \mathrm{Tol}_{\text {correction }}
$$

is satisfied. We use Tol $\mathrm{C}_{\text {correction }}=10^{-11}$ in all our computations.

For each time step a grid hierarchy is obtained either by uniform refinement or according to the adaptive coarsening and refinement strategy described in Subsection 3.2.3.

The initial iterate for the algebraic Schur-Newton solver is derived by nested iteration, i.e., on each refinement level an initial iterate is obtained by nodal interpolation of the final iterate from the preceding one. On the first refinement level, the initial iterate is obtained by nodal interpolation of the final approximation in the preceding time step. For the first time step, the continuous initial conditions are interpolated to the initial grid $\mathcal{T}^{\text {old }}$.

All numerical experiments were conducted using the DUNE (Distributed and Unified Numerics Environment) framework and the DUNE-modules dune-subgrid and dune-tnnmg (cf. [7, 8, 32]).

5.1. Experimental order of convergence. In order to numerically assess the spatial discretization error of the finite element discretization stated in Problem 3.2, we consider the multi-phase Penrose-Fife Problem 2.1, with $\Omega=(0,2)^{2} \subset \mathbb{R}^{2}$, $M=5$ phases of which only the liquid and one solid phase is present, and the following parameters

$$
\begin{aligned}
& \varepsilon=6 \cdot 10^{-2}, \quad c_{v}=1, \quad q=0, \quad h_{c}=0, \\
& \kappa=1, \quad \beta=1, \quad L_{1}=0, \quad L_{\alpha}=2, \alpha=2, \ldots, M \text {. }
\end{aligned}
$$

We select the initial temperature $\theta^{0}=0.5$. The initial phase field $\phi^{0}$ is given by

$$
\phi_{2}^{0}(x)= \begin{cases}1 & \text { if } d(x)<0.5 \\ \left|\frac{1}{2} \cos (5 \pi(d(x)-0.5))+0.5\right| & \text { if } 0.5 \leq d(x)<0.7 \\ 0 & \text { else }\end{cases}
$$

where $d(x)$ stands for the Euclidean distance from $x$ to $(1,1), \phi_{1}^{0}(x)=1-\phi_{2}^{0}(x, t)$, and $\phi_{\alpha}^{0}=0$ for $\alpha=3,4,5$, as depicted in Figure 1. We select the uniform time step size $\tau=5 \cdot 10^{-4}$. A sequence $\mathcal{T}_{0}, \ldots, \mathcal{T}_{10}$ of grids is obtained by uniform refinement of $\mathcal{T}_{0}$ consisting of a partition of $\Omega$ into two triangles.

Figure 2 shows the approximate discretization error in the first time step plotted over the mesh size $h_{j}, j=2, \ldots, 9$. The exact error is approximated by $e_{\phi}=\phi_{\mathcal{T}}-\phi^{*}$ and $e_{\theta}=\theta_{\mathcal{T}}-\theta^{*}$, with approximations $\phi^{*}$ and $\theta^{*}$ obtained from $\mathcal{T}_{10}$. Our results suggest optimal order $O(h)$ of the discretization error $\|e\|=\left\|e_{\phi}\right\|_{\phi}+\left\|e_{\theta}\right\|_{\theta}$.

We next investigate the convergence properties of the non-smooth Schur-Newton method as applied to the discrete saddle point problem in the first time step. Figure 3 depicts the number $\nu_{\max }$ of iteration steps needed until the stopping criterion (48) is satisfied plotted over $N=\operatorname{dim} \mathcal{S}_{j}$, where $\mathcal{S}_{j}$ is the finite element space associated 


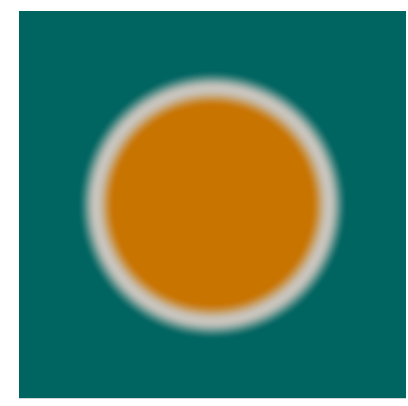

FiguRE 1. Initial phase field: A circular solid phase (orange) in a liquid environment (teal).

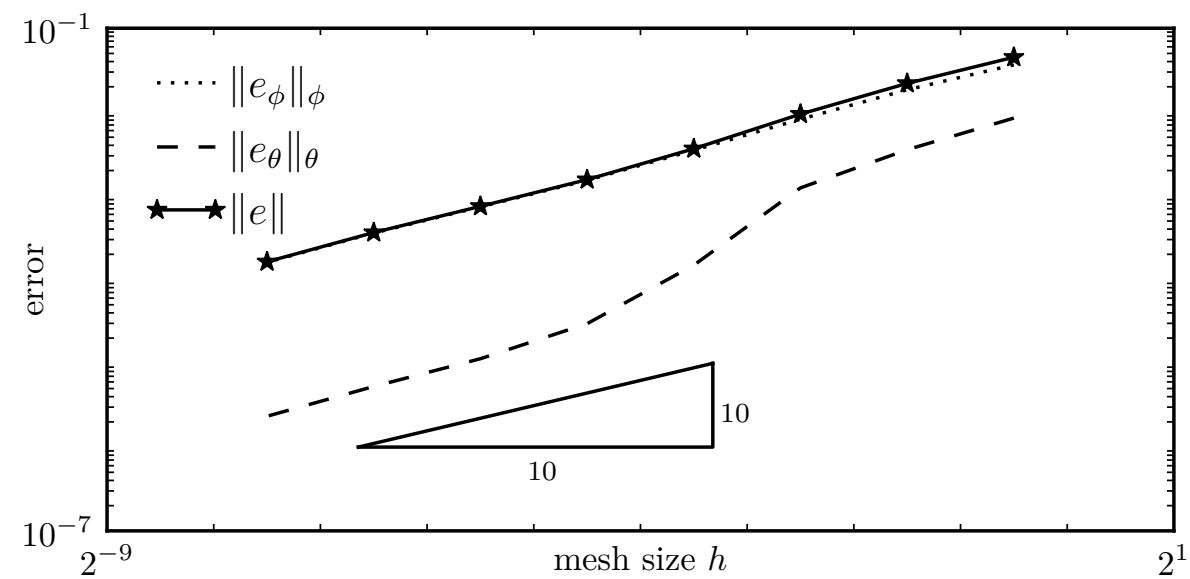

Figure 2. Discretization error $\|e\|=\left\|e_{\phi}\right\|_{\phi}+\left\|e_{\theta}\right\|_{\theta}$ and its components $\left\|e_{\phi}\right\|_{\phi}$ and $\left\|e_{\theta}\right\|_{\theta}$ over mesh size.

with $\mathcal{T}_{j}$. The results indicate mesh independence of the Schur-Newton iteration. While up to $\nu_{\max }=17$ iteration steps are required on coarser levels, only $\nu_{\max } \leq 7$ steps are needed once the diffuse interface is properly resolved by sufficiently fine grids. This is in accordance with previous computations with multi-component Cahn-Hilliard systems [29].

We also computed the approximate solution for the first 500 time steps utilizing the grid $\mathcal{T}_{7}$ obtained by seven uniform refinements to illustrate the evolution of the approximate entropy as depicted in Figure 4.

5.2. Evolution of energy and entropy. In order to illustrate the equilibration of energy in terms of latent heat and temperature and the evolution of entropy, we consider the multi-phase Penrose-Fife Problem 2.1 on the unit square $\Omega=(0,1)^{2} \subset$ $\mathbb{R}^{2}$ with $M=5$ phases of which only the liquid and one solid phase are present, and the parameters

$$
\begin{aligned}
& \varepsilon=8 \cdot 10^{-2}, \quad c_{v}=1, \quad q=0, \quad h_{c}=0, \\
& \kappa=1, \quad \beta=1, \quad L_{1}=0, \quad L_{\alpha}=2, \alpha=2, \ldots, 5 \text {. }
\end{aligned}
$$




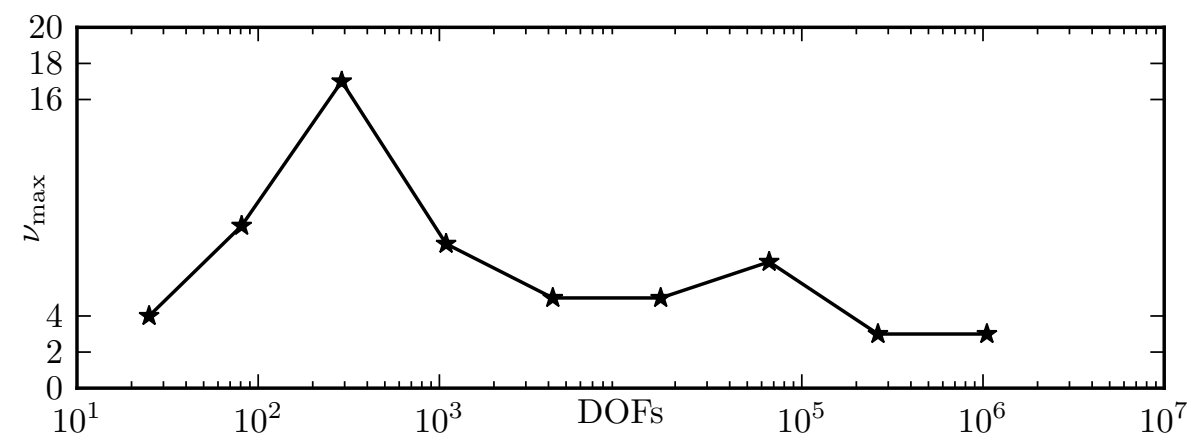

FiguRE 3. Number of Schur-Newton iterations $\nu_{\max }$ needed to solve Problem 3.2 over $N$.

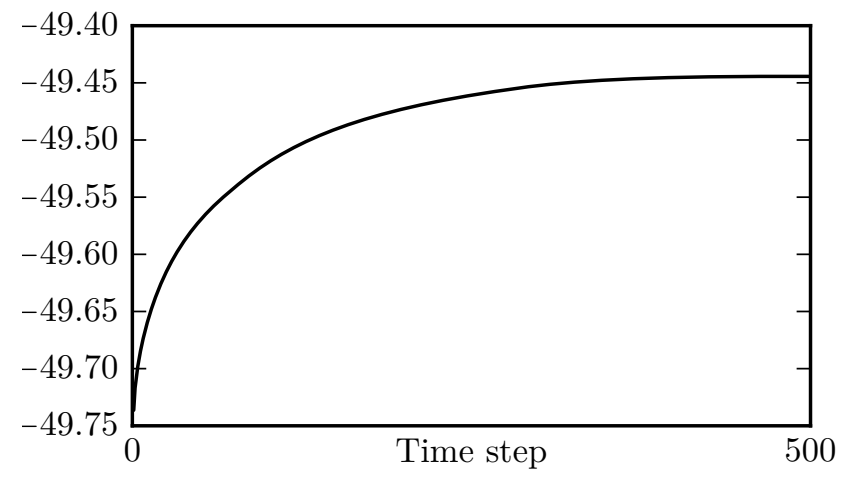

Figure 4. Approximate entropy over time steps.

We choose an initial configuration with two phases (liquid and solid) and a planar interface according to

$$
\phi_{2}^{0}\left(x_{1}, x_{2}\right)= \begin{cases}1 & \text { if } x_{1}>x^{\star}+0.1 \\ 10\left(x_{1}-x^{\star}\right) & \text { if } x^{\star}+0.1 \geq x_{1}>x^{\star} \\ 0 & \text { if } x^{\star} \geq x_{1},\end{cases}
$$

$\phi_{1}^{0}=1-\phi_{2}^{0}$, and $\phi_{\alpha}^{0}=0, \alpha=3,4,5$. The parameter $x^{\star}$ and constant initial temperature $\theta^{0}=\theta^{\star}$ will be fixed later.

We select the time step size $\tau=5 \cdot 10^{-3}$. The grid $\mathcal{T}$ is obtained by eight uniform refinements of an initial partition of $\Omega$ into two triangles.

The evolution of temperature is illustrated in terms of its maximal variation

$$
\theta_{d}^{n}=\max _{x \in \Omega} \theta^{n}(x)-\min _{x \in \Omega} \theta^{n}(x), \quad n=1, \ldots, 500,
$$

and the average

$$
\theta_{m}^{n}=\frac{1}{2}\left(\max _{x \in \Omega} \theta^{n}(x)+\min _{x \in \Omega} \theta^{n}(x)\right), \quad n=1, \ldots, 500,
$$

of its extremal values. The parameter $x^{\star}$ and constant initial temperature $\theta^{0}=\theta^{\star}$ are set to $x^{\star}=0.8$ and $\theta^{\star}=0.2^{-1}$ in our first experiment and to $x^{\star}=0.2$ and $\theta^{\star}=1.5^{-1}$ in our second experiment. The corresponding two evolutions are illustrated in Figure 5 and Figure 6, respectively. Both figures show several time steps of the phase field in the left picture. As the solution is constant in vertical 

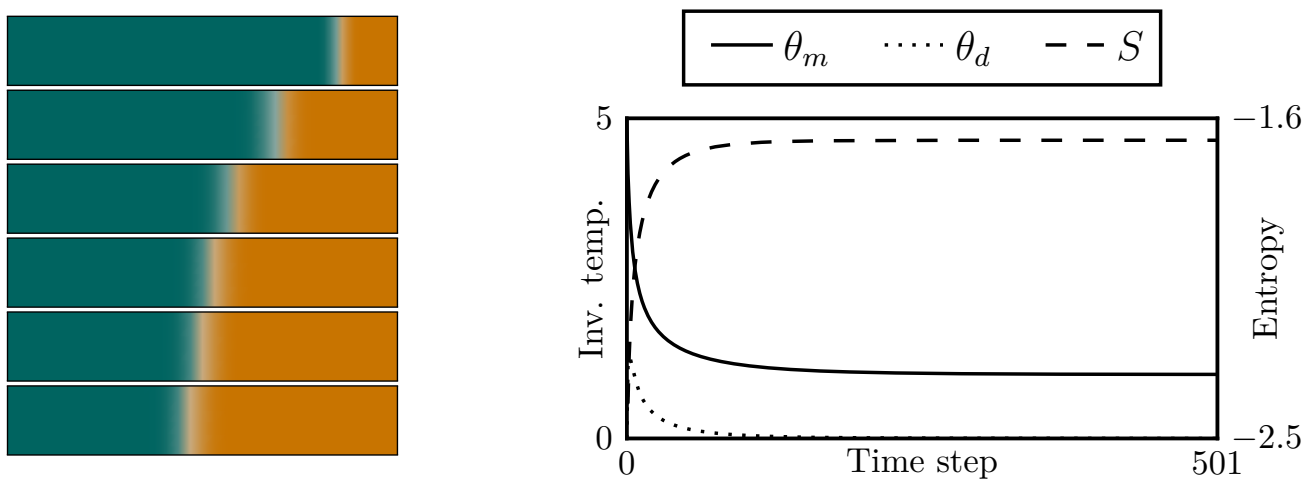

FIGURE 5. Solidification induced by latent heat. Left: Evolution of phases by means of approximations at the time steps 0,40 , 80, 120, 160, 500. Right: Evolution of inverse temperature $\theta$ and approximate entropy $S$.
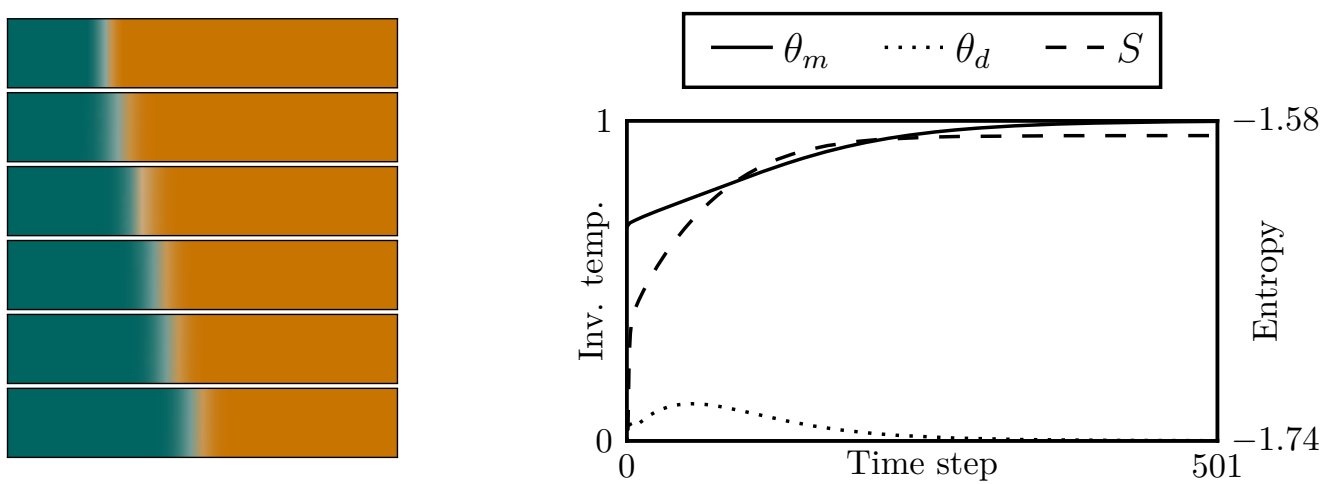

Figure 6. Melting induced by latent heat. Left: Evolution of phases by means of approximations at the time steps $0,40,80$, 120, 160, 500. Right: Evolution of inverse temperature $\theta$ and approximate entropy $S$.

direction only a cut-out is shown. The picture on the right shows the evolution of temperature in terms of $\theta_{m}^{n}$ and $\theta_{d}^{n}$ and of the entropy $S=\hat{S}\left(\theta^{n}, \phi^{n}\right)$ (cf. (25)) approximated by numerical quadrature.

In the first experiment we observe a growth of the initial solid grain that slows down continuously due to intrinsic specimen heating by solidification. Conversely, the shrinking of the initial grain observed in the second experiment slows down due to intrinsic cooling by melting. In both experiments, absorption or release of latent heat is driving the approximate temperature $\frac{1}{\theta^{n}}$ towards the melting temperature $T=1$ at equilibrium. Both cases exhibit a monotonically increasing entropy.

\section{A Liquid phase CRYstallization PROCEss}

Liquid phase crystallization (LPC) is an emerging technology to produce silicon thin film solar cells with advanced photoelectronic properties that enable high efficiency devices. In an LPC process, silicon is deposited on a substrate and then is swept over with a heat source for local melting and subsequent recrystallization to 

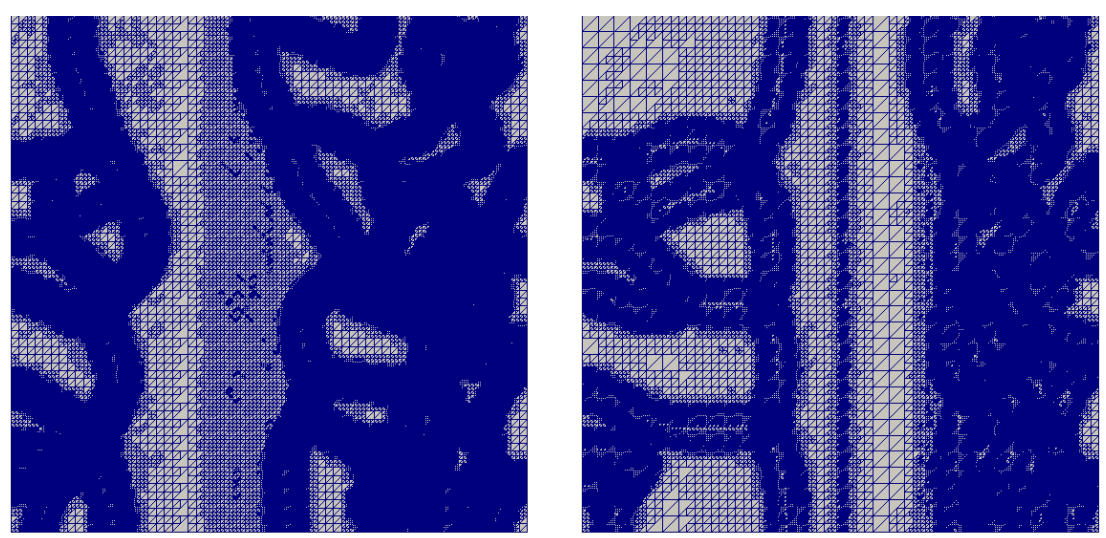

Figure 7. Adaptively refined grid for time step 5 (left) and 100 (right).

coarser, photoelectronically beneficial structures. Optimization of parameters like speed, shape or intensity of the heat source for various semi-conducting materials is the subject of current experimental research, cf., e.g., [2, 23, 41].

Mathematical modelling of LPC processes can be performed in the framework of multi-phase field models presented in Section 2. To this end, we consider the thin-film approximation Problem 2.2 on $\Omega=(0,2)^{2}$ with $M=5$ phases with $\phi_{\alpha}$, $\alpha=2, \ldots M$, representing different crystal structures and the parameters

$$
\begin{aligned}
& \varepsilon=5 \cdot 10^{-2}, \quad c_{v}=1, \quad T_{\Gamma}=0.1, \quad T_{\alpha}=1, \alpha=2, \ldots, M, \\
& h_{c}^{\prime}=5 \cdot 10^{2}, \quad \kappa=1, \quad L_{1}=0, \quad L_{\alpha}=1, \alpha=2, \ldots, M .
\end{aligned}
$$

In order to prescribe a slower solid-solid interface evolution in comparison the to solid-liquid interfaces, we now choose a solution-dependent kinetic coefficient $\beta=\left(\beta_{\alpha}(\phi, \nabla \phi)\right)_{\alpha=1}^{N}$ according to

$$
\beta_{\alpha}\left(\phi_{1}, \ldots, \phi_{M}, \nabla \phi_{1}, \ldots, \nabla \phi_{M}\right)= \begin{cases}100 & \left|\phi_{1} \nabla \phi_{\alpha}-\phi_{\alpha} \nabla \phi_{1}\right|<10^{-5} \\ 1 & \text { else. }\end{cases}
$$

The heat source is represented by

$$
\begin{aligned}
q(\theta, x, t) & =q_{\max }(\theta) \cdot \exp \left(-\frac{\left|x_{1}-q_{p}(t)\right|}{2 q_{w}^{2}}\right), \\
q_{\max }(\theta) & =\frac{1}{\theta} h_{q}\left(1-\frac{\theta_{q}}{\theta}\right)+h_{c}^{\prime}\left(\frac{1}{\theta}-T_{\Gamma}\right)
\end{aligned}
$$

and we select the parameters

$$
q_{p}(t)=0.9+1.5 t, \quad q_{w}=0.2, \quad \theta_{q}=\frac{1}{8} \quad h_{q}=5 \cdot 10^{2} .
$$

Notice that the consistency statements of Proposition 2.1 and 3.1 cannot be applied in this case, because $q \neq 0$ and because the coefficient $\beta$ depends on $\phi$. It is unclear if similar results can be shown for solution dependent coefficients.

Observe that the heat source peaks at $x_{1}=q_{p}(t)$ and is moving across the device from left to right with constant speed. The initial temperature is given by $\theta^{0}=10=\frac{1}{T^{0}}=\frac{1}{0.1}$ and the initial phase configuration $\phi^{0}$ is depicted in the upper left picture of Figure 8 with teal color representing the liquid phase. We choose an initial value that already prescribes a local liquid phase, because this simplifies to exclude superheating effects such as instantaneous global melting of the whole material.

We select the time step size $\tau=2 \cdot 10^{-3}$. In each time step, we construct a sequence of locally refined meshes $\mathcal{T}_{0}, \ldots, \mathcal{T}_{J}$ using the adaptive refinement algorithm 

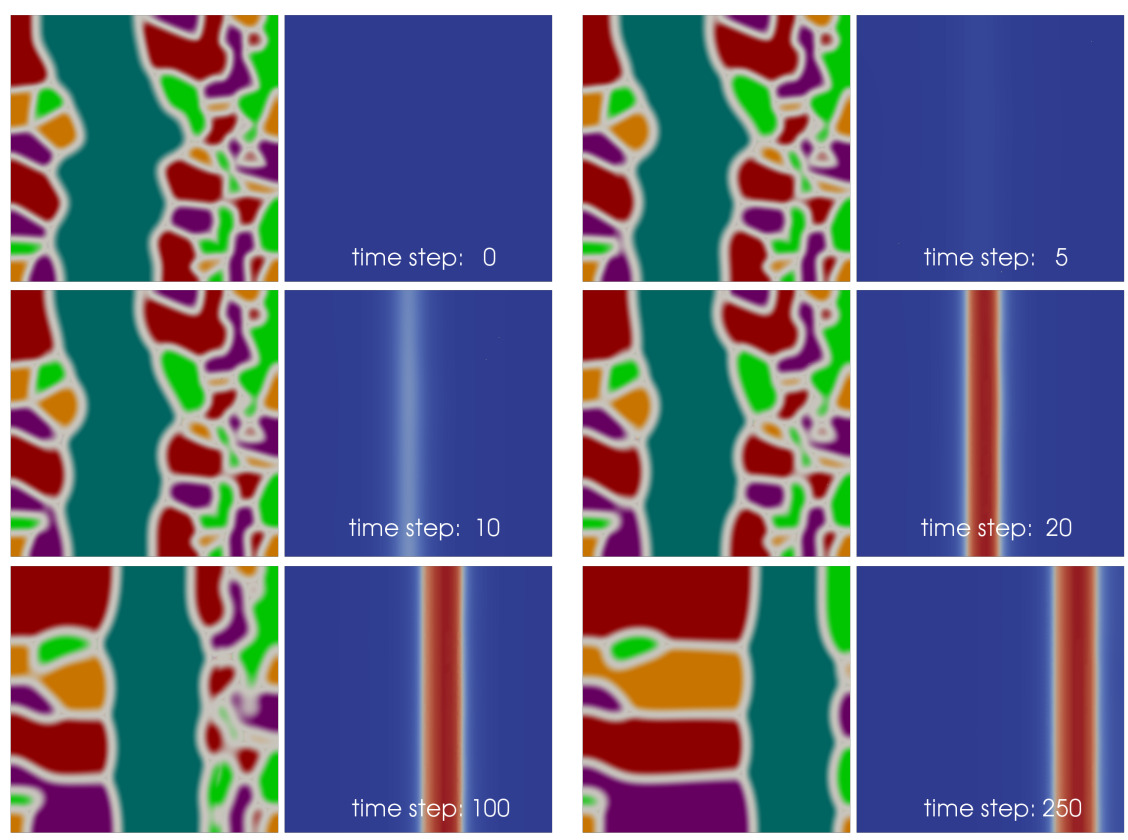

FiguRE 8. Initial distribution of phases and temperature (top left) and approximate distribution of phases and temperature for the time steps 5, 10, 20, 100, 250.
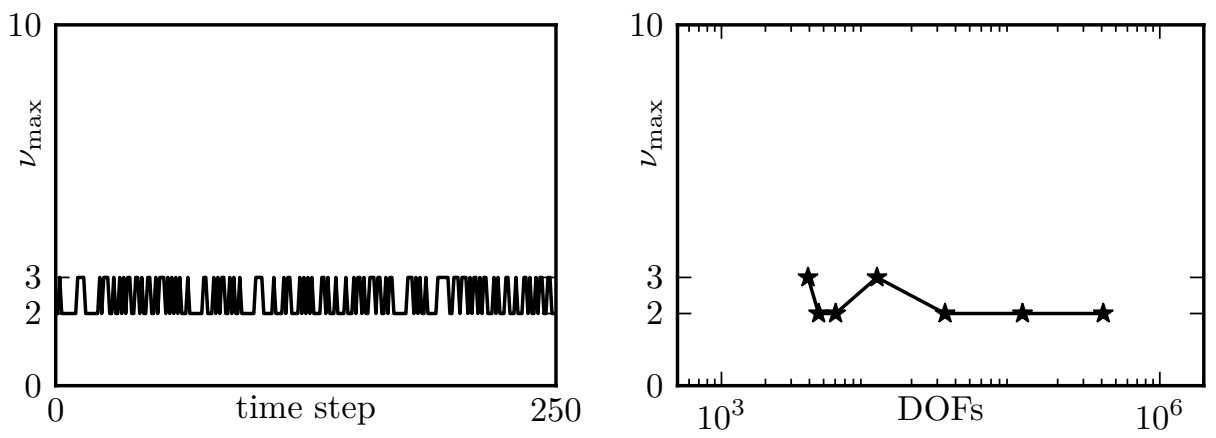

FiguRE 9. Robustness of Schur-Newton convergence. Left: $\nu_{\max }$ corresponding to the final mesh over time steps. Right: $\nu_{\max }$ corresponding to different adaptively refined meshes over degrees of freedom $N$ for the fixed time step 5 .

described in Subsection 3.2.3. The derefinement and refinement parameters are selected as follows

$$
j_{\text {min }}=2, \quad \text { Tol }_{\text {derefine }}=10^{-6}, \quad \rho=0.9, \quad \operatorname{Tol}_{\text {adapt }}=8 \cdot 10^{-3} .
$$

In the first time step, we start with an initial grid $\mathcal{T}^{\text {old }}$ obtained by eight uniform refinements of an initial partition of $\Omega$ into two triangles. The final mesh $\mathcal{T}$ for time steps 5 and 100 is depicted in Figure 7. In both cases, the mesh is obtained by 6 adaptive refinement steps after coarsening.

Figure 8 shows the (approximate) evolution of phases and temperature. For each of the time steps $0,5,10,20,100,250$ the left picture depicts the liquid 
and the different crystalline phases while the right picture shows the temperature distribution. The liquid phase adapts to the shape of the heat source in course of the evolution. As the heat source travels on, the right hand crystalline phases start melting, while recrystallization occurs on the left solid-liquid interfaces, because the local temperature drops below melting temperature. Note that recrystallization leads to coarser grain structures which is a characteristic feature of LPC.

To briefly highlight the efficiency of the Schur-Newton method with nested iteration, the number of Schur-Newton steps $\nu_{\max }$ required on the finest mesh is plotted over the time step in the left of Figure 9. We observe that this number does not exceed 3 in any time step. Mesh independence is illustrated by the number of Schur-Newton steps over $N=\operatorname{dim} \mathcal{S}_{j}$ in time step 5 .

\section{ACKNOWLEDGMENT}

The work was supported by the Helmholtz Virtual Institute HVI-520 "Microstructure Control for Thin-Film Solar Cells", the Einstein Foundation Berlin via the research center MATHEON, and by the Sino-German Science Center (grant id 1228) on the occasion of the Chinese-German Workshop on Computational and Applied Mathematics in Augsburg 2015.

\section{REFERENCES}

[1] H. W. Alt and I. Pawlow. A mathematical model of dynamics of non-isothermal phase separation. Physica D: Nonlinear Phenomena, 59(4):389-416, 1992.

[2] D. Amkreutz, J. Müller, M. Schmidt, T. Hänel, and T. Schulze. Electron-beam crystallized large grained silicon solar cell on glass substrate. Progress in Photovoltaics: Research and Applications, 19(8):937-945, 2011.

[3] C. Baiocchi and A.-C. Capelo. Variational and quasivariational inequalities: Applications to free boundary problems. Wiley, 1984.

[4] R. E. Bank and R. K. Smith. A posteriori error estimates based on hierarchical bases. SIAM J. Numer. Anal., 30:921-935, 1993.

[5] J. W. Barrett and J. F. Blowey. Finite element approximation of a model for phase separation of a multi-component alloy with non-smooth free energy. Numerische Mathematik, 77(1):1-34, 1997.

[6] S. Bartels. Numerical Methods for Nonlinear Partial Differential Equations, volume 47. Springer, 2015.

[7] P. Bastian, M. Blatt, A. Dedner, C. Engwer, R. Klöfkorn, R. Kornhuber, M. Ohlberger, and O. Sander. A generic interface for adaptive and parallel scientific computing. Part II: Implementation and tests in DUNE. Computing, 82(2-3):121-138, 2008.

[8] P. Bastian, M. Blatt, A. Dedner, C. Engwer, R. Klöfkorn, M. Ohlberger, and O. Sander. A generic interface for adaptive and parallel scientific computing. Part I: Abstract framework. Computing, 82(2-3):103-119, 2008

[9] J. Bey. Simplicial grid refinement: On Freudenthal's algorithm and the optimal number of congruence classes. Numer. Math., 85:1-29, 2000.

[10] L. Blank, H. Garcke, L. Sarbu, and V. Styles. Nonlocal Allen-Cahn systems: analysis and a primal-dual active set method. IMA J. Numer. Anal., 2013.

[11] L. Blank, H. Garcke, L. Sarbu, and V. Styles. Primal-dual active set methods for Allen-Cahn variational inequalities with nonlocal constraints. Numerical Methods for Partial Differential Equations, 29(3):999-1030, 2013.

[12] J. F. Blowey, M. I. M. Copetti, and C. M. Elliott. Numerical analysis of a model for phase separation of a multi-component alloy. IMA J. Numer. Anal., 16(1):111-139, 1996.

[13] J. F. Blowey and C. M. Elliott. The Cahn-Hilliard gradient theory for phase separation with non-smooth free energy part I: Mathematical analysis. European J. Appl. Math., 2:233-280, 1991.

[14] F. Bornemann, B. Erdmann, and R. Kornhuber. Adaptive multilevel methods in three space dimensions. Int. J. Numer. Meth. Engrg., 36:3187-3203, 1993.

[15] F. A. Bornemann. An Adaptive Multilevel Approach for Parabolic Equations in Two Space Dimensions. PhD thesis, Freie Universität Berlin, 1991.

[16] F. A. Bornemann, B. Erdmann, and R. Kornhuber. A posteriori error estimates for elliptic problems in two and three space dimensions. SIAM J. Numer. Anal., 33:1188-1204, 1996. 
[17] M. Brokate and J. Sprekels. Hysteresis and Phase Transitions. Springer, 1996.

[18] J. Crank. Free and moving boundary problems. Clarendon press Oxford, 1984.

[19] K. Deckelnick, G. Dziuk, and C. M. Elliott. Computation of geometric partial differential equations and mean curvature flow. Acta numerica, 14:139-232, 2005.

[20] P. Deuflhard and F. A. Bornemann. Numerische Mathematik 2. Walter de Gruyter, Berlin, 3. edition, 2008.

[21] P. Deufhard, P. Leinen, and H. Yserentant. Concepts of an adaptive hierarchical finite element code. IMPACT Comput. Sci. Engrg., 1:3-35, 1989.

[22] W. Dörfler. A convergent adaptive algorithm for Poisson's equation. SIAM J. Numer. Anal., 33:1106-1124, 1996.

[23] B. Eggleston, S. Varlamov, J. Huang, R. Evans, J. Dore, and M. A. Green. Large grained, low defect density polycrystalline silicon on glass substrates by large-area diode laser crystallisation. In MRS Proceedings, volume 1426, pages 251-256. Cambridge Univ Press, 2012.

[24] H. Garcke, B. Nestler, and B. Stinner. A diffuse interface model for alloys with multiple components and phases. SIAM J. Appl. Math., 64:775-799, 2004.

[25] C. Gräser. Convex Minimization and Phase Field Models. PhD thesis, Freie Universität Berlin, 2011.

[26] C. Gräser and R. Kornhuber. Nonsmooth Newton methods for set-valued saddle point problems. SIAM J. Numer. Anal., 47(2):1251-1273, 2009.

[27] C. Gräser, R. Kornhuber, and U. Sack. On hierarchical error estimators for time-discretized phase field models. In G. Kreiss, P. Lötstedt, A. Målqvist, and M. Neytcheva, editors, Numerical Mathematics and Advanced Applications 2009, pages 397-405. Springer, 2010.

[28] C. Gräser, R. Kornhuber, and U. Sack. Time discretizations of anisotropic Allen-Cahn equations. IMA Journal of Numerical Analysis, 33(4):1226-1244, 2013.

[29] C. Gräser, R. Kornhuber, and U. Sack. Nonsmooth Schur-Newton methods for multicomponent Cahn-Hilliard systems. IMA Journal of Numerical Analysis, 2014.

[30] C. Gräser, R. Kornhuber, and U. Sack. Numerical simulation of coarsening in binary solder alloys. Comp. Mater. Sci., 93:221-233, 2014.

[31] C. Gräser, U. Sack, and O. Sander. Truncated nonsmooth Newton multigrid methods for convex minimization problems. In Domain Decomposition Methods in Science and Engineering XVIII, pages 129-136. Springer, 2009.

[32] C. Gräser and O. Sander. The dune-subgrid module and some applications. Computing, 86(4):269-290, 2009.

[33] C. Gräser and O. Sander. Truncated nonsmooth Newton multigrid methods for simplexconstrained minimization problems. Preprint 384, IGPM Aachen, 2014. submitted.

[34] M. Holst, J. Ovall, and R. Szypowski. An efficient, reliable and robust error estimator for elliptic problems in $\mathbf{R}^{3}$. Appl. Num. Math., 61:675-695, 2011.

[35] R. H. W. Hoppe and R. Kornhuber. Adaptive multilevel-methods for obstacle problems. SIAM J. Numer. Anal., 31(2):301-323, 1994.

[36] D. Kinderlehrer and G. Stampacchia. An introduction to variational inequalities and their applications, volume 31. SIAM, 1980.

[37] O. Klein. Existence and Approximation Results for Phase-Field Systems of Penrose-Fife Type and Stefan Problems. Shaker, 1997.

[38] R. Kornhuber. A posteriori error estimates for elliptic variational inequalities. Comput. Math. Appl., 31:49-60, 1996.

[39] R. Kornhuber and R. Krause. Robust multigrid methods for vector-valued Allen-Cahn equations with logarithmic free energy. Comput. Vis. Sci., 9:103-116, 2006.

[40] R. Kornhuber and Q. Zou. Efficient and reliable hierarchical error estimates for the discretization error of elliptic obstacle problems. Math. Comp., 80:69-88, 2011.

[41] S. Kühnapfel, S. Gall, B. Rech, and D. Amkreutz. Towards monocrystalline silicon thin films grown on glass by liquid phase crystallization. Solar Energy Materials and Solar Cells, 140:86-91, 2015.

[42] B. Nestler, F. Wendler, M. Selzer, B. Stinner, and H. Garcke. Phase-field model for multiphase systems with preserved volume fractions. Physical Review E, 78(1):011604, 2008.

[43] R. Nürnberg. Numerical simulations of immiscible fluid clusters. Appl. Numer. Math., 59(7):1612-1628, 2009.

[44] J. M. Ortega and W. C. Rheinboldt. Iterative Solution of Nonlinear Equations in Several Variables. Academic Press, New York, 1970.

[45] O. Penrose and P. C. Fife. Thermodynamically consistent models of phase-field type for the kinetic of phase transitions. Physica D: Nonlinear Phenomena, 43(1):44-62, 1990.

[46] K. G. Siebert and A. Veeser. A unilaterally constrained quadratic minimization with adaptive finite elements. SIAM J. Optim., 18:260-289, 2007. 
[47] B. Stinner. Weak solutions to a multi-phase field system of parabolic equations related to alloy solidification. Adv. Math. Sc. Appl., 17(2):589-638, 2007.

[48] A. Visintin. Models of phase transitions, volume 28. Springer Science \& Business Media, 2012.

[49] O. C. Zienkiewicz, J. P. de S. R. Gago, and D. W. Kelly. The hierarchical concept in finite element analysis. Computers \& Structures, 16:53-65, 1983.

[50] Q. Zou, A. Veeser, R. Kornhuber, and C. Gräser. Hierarchical error estimates for the energy functional in obstacle problems. Numer. Math., 117(4):653-677, 2011.

Carsten Gräser, Freie Universität Berlin, Institut für Mathematik, Arnimallee 6, D - 14195 Berlin, Germany

E-mail address: graeser@math.fu-berlin.de

Max Kahnt, Freie Universität Berlin, Institut für Mathematik, Arnimallee 6, D 14195 Berlin, Germany

E-mail address: max.kahnt@math.fu-berlin.de

Ralf Kornhuber, Freie Universität Berlin, Institut für Mathematik, Arnimallee 6, D - 14195 Berlin, Germany

E-mail address: kornhuber@math.fu-berlin.de 